\title{
Is candidiasis the true cause of vulvovaginal irritation in women with diabetes mellitus?
}

\author{
B R Rowe, M N Logan, I Farrell, A H Barnett
}

\begin{abstract}
Vulvovaginitis is common in diabetic women and is often treated with antifungal agents on the assumption that the causative organism is Candida albicans. In a survey of 100 consecutive diabetic women attending a diabetes clinic 36 had complained to their general practitioner about vulvovaginal irritation during the past three years and 26 were treated with antifungal agents without a vaginal examination or swabs being taken. In a separate study 27 post-menopausal women with non-insulin dependent diabetes and symptoms of vulvovaginitis were investigated. The organisms cultured were: Candida albicans $(n=6), \beta$ haemolytic streptococci $(n=14)$, Gardnerella vaginalis $(n=2)$, Staphylococcus aureus $(\mathbf{n}=2)$, Streptococcus milleri $(\mathbf{n}=1)$, Streptococcus faecalis $(\mathrm{n}=1)$, Klebsiella oxytoca $(n=1)$, no organisms $(n=3)$. Where a bacterial organism was isolated symptoms resolved in all but one case with appropriate antibiotic treatment.

It is recommended that the practice of initiating antifungal treatment without taking high vaginal swabs should be reviewed and treatment should be given specifically rather than empirically.
\end{abstract}

Vulvovaginal candidiasis affects about $75 \%$ of all adult women at some stage in their lives. ${ }^{1}$ Several related factors, including diabetes mellitus, have been identified. ${ }^{2}$ Uncontrolled diabetes with glycosuria and increased glucose concentrations in vaginal secretions may precipitate symptomatic vaginitis presumed to be due to colonisation with Candida albicans. ${ }^{3}$

Women with diabetes are often treated with antifungal agents without a vaginal examination or swabs being taken. We report a survey of the extent of the problem of vulvovaginitis in our diabetic clinic and an investigation of the causative organisms.

\footnotetext{
Department of Medicine, East Birmingham Hospital and University of Birmingham, B9 5ST B R Rowe, A H Barnett Department of Bacteriology M N Logan, I Farrell Correspondence to: Dr A H Barnett Accepted for publication 15 March 1990
}

\section{Methods}

One hundred consecutive women with noninsulin dependent diabetes mellitus (NIDDM) attending the diabetic clinic were asked to fill in a questionnaire (group 1). Each patient was asked about duration, type and treatment of diabetes, and whether they had had any prolonged episodes of vaginal irritation in the past three years. If they had consulted their general practitioner about their vulvovaginitis they were asked if they had been given an internal examination before being given any treatment.

In a separate study 27 consecutive female patients with NIDDM with symptoms of vaginal or vulval pruritis and discharge were studied (group 2). A gynaecological history was taken and each patient had a high vaginal swab and mid-stream urine specimen at three consecutive weekly visits. The specimens were examined for the presence of clinically important microbiological pathogens, and the organisms were identified by conventional bacteriological and fungal methods. Duration of symptoms, discharge, and excoriation of the vulva were noted.

If antibiotic treatment was begun the investigations were repeated after a three week interval.

Blood was taken for estimation of glycosylated haemoglobin $\left(\mathrm{HbA}_{1}\right)$ and serum glucose. $\mathrm{HbA}_{1}$ was measured by affinity chromatography (CV $2 \%$, reference range 5$9 \%$ ). Written, informed consent was obtained for the separate study. The study was approved by the local ethical committee.

\section{Results}

Thirty six of the 100 patients attending our clinic had seen their general practitioner about vulvovaginal irritation. Twenty six of these had been treated with antifungal agents without a vaginal examination or swabs being taken (group 1).

The mean age of group 2 subjects was 64 years, range $49-88$ years, and they were receiving oral hypoglycaemic agents. Mean duration of symptoms was $7 \cdot 1$ weeks before their routine outpatient appointment; no patient was referred specifically because of vaginal irritation.

The results of the high vaginal swab cultures are shown in the table. Six women $(16.6 \%)$ had vaginal candidiasis, in $14(51.8 \%)$ a $\beta$ haemolytic Streptococcus (groups A, B, C and G) was cultured as the sole organism or in

Result of high vaginal swab cultures in symptomatic patients
Candida albicans

Group A $\beta$ haemolytic Streptocuccus

Group B $\beta$ haemolytic Streptococcus

Group $\mathrm{C}$ or $\mathrm{G} \beta$ haemolytic Streptococcus

Group B $\beta$ haemolytic Streptococcus $+C$ albicans Group $C \beta$ haemolytic Streptococcus $+G$ vaginalis 1 Group $C \beta$ haemolytic Streptococcus $+G$ vaginalis Group B $\beta$ haemolytic Streptococcus $+S$ faecalis Staphylococcus aureus
Klebsiella oxytoca

Streptococcus milleri

Streptococcus milleri
No pathogens isolated Gardnerella vaginalis 
conjunction with either $S$ faecalis $(\mathbf{n}=1), G$ vaginalis $(\mathrm{n}=1)$, or $C$ albicans $(\mathrm{n}=1)$. The other patients had $G$ vaginalis $(\mathrm{n}=1), S$ aureus $(\mathrm{n}=2), S$ milleri $(\mathrm{n}=1)$ and Klebsiella oxytoca $(\mathrm{n}=1)$. Three symptomatic patients had no obvious cause for their irritation. Six patients had a concomitant Escherichia coli urinary tract infection, three of these in association with streptococcal vaginitis. The one patient in whom $K$ oxytoca was isolated from a high vaginal specimen also had a Klebsiella urinary tract infection which was successfully treated with one antibiotic. All other patients with urinary tract infection required two different antibiotics specific to each pathogen.

All but one patient in the group in whom bacteria were cultured from the vagina had complete resolution of symptoms after antibiotic treatment specific to the cultured organism. One patient who had been prescribed several courses of antifungal agents over the course of six months had a streptococcal infection. Her symptoms resolved with appropriate antibiotic treatment. Another patient in whom $S$ aureus was cultured was found to have vaginitis with oedema and pronounced excoriation of the vulva. Her blood glucose concentration was $20.4 \mathrm{mmol} / 1$ and it was felt that she needed insulin. When antibiotic treatment was given her symptoms resolved, her blood glucose stabilised, and she was able to continue to have oral hypoglycaemic agents to control her diabetes.

There was a trend towards a higher mean blood glucose (mean 15.3 (SD)(4.3) v 12.2(5.1) mmol/1) and $\mathrm{HbA}_{1}(12 \cdot 1$ (3.5) v $9 \cdot 7$ (3.0) \%) in the patients with vaginal candidiasis compared with those with streptococcal infection, but formal statistical analysis was not done because of the small numbers in the former group.

\section{Discussion}

Vulvovaginitis is common in diabetic women and has typically been attributed to Candida albicans. ${ }^{3}$ In this series of 27 women with symptoms of vulvovaginitis $C$ albicans was cultured in only six cases. In over $50 \%$ of cases a $\beta$ haemolytic Streptococcus was isolated. Of these, group $B$ was the most common, being found in 11 of the 14 cases. Groups A, C, and G were isolated from the remaining three patients. Other organisms cultured included $S$ milleri, $S$ faecalis, and $S$ aureus.

Little is known about the epidemiological factors that are related to the presence of these organisms in the genital tract. In the absence of other vaginal pathogens to explain symptoms a culture of Lancefield group A $\beta$ haemolytic Streptococcus would be considered clinically important whereas the presence of groups B, C, and $G$ is usually thought to reflect colonisation from the gastrointestinal tract. ${ }^{4}$ Vaginal colonisation with the Lancefield group B $\beta$ haemolytic Streptococcus has been reported in $18 \%$ of 499 asymptomatic college women. ${ }^{5}$ The subjects were sexually active young women and colonisation was thought to result from sexual contact. Several studies have documented the carriage rate of $\beta$ haemolytic Streptococcus in pregnant women, and it may be as high as $30 \%{ }^{4}$ In our study only five subjects were sexually active, and then very infrequently. There are no studies documenting the carriage rates of $\beta$ haemolytic Streptococcus in postmenopausal or diabetic women.

The pathogenic role of $G$ vaginalis in vulvovaginitis is often reported, but $S$ milleri and $S$ faecalis are considered to be normal commensals in younger women. Older, postmenopausal women have decreased oestrogen production with thinning and inactivity of vaginal epithelium, together with a reduction in acidity and a rise in vaginal $\mathrm{pH}$. In certain circumstances the combination of these factors may result in the organisms becoming pathogenic. In diabetics poor glycaemic control is also thought to result in impaired mobilisation and action of phagocytic cells, particularly polymorphonuclear leucocytes, ${ }^{6}$ resulting in decreased ability to resist infection from opportunistic organisms. The clinical importance of the organism cultured from this symptomatic group remains doubtful, but it is interesting to note that all but one patient had a rapid symptomatic response to treatment with appropriate antimicrobial agents. The patients had similar symptoms irrespective of the cultured organism. A possible discriminating factor was the common complaint of nocturnal irritation in those patients infected with $\beta$ haemolytic Streptococci. The six patients who had $C$ albicans tended to have worse glycaemic control.

In conclusion, $C$ albicans was not found as frequently as expected in diabetic women with vulval irritation. The practice of initiating antifungal treatment without taking high vaginal swabs should therefore be reviewed. In this elderly non-insulin dependent diabetic group antimicrobial treatment directed at organisms usually regarded as commensals led to a speedy resolution of symptoms.

BR is supported by ICI Pharmaceuticals.

1 Sobel JD. Epidemiology and pathogenesis of recurrent vulvovaginal candidiasis. Am J Obstet Gynecol 1985; 152:924-35.

2 De Costa E. Infections of the vagina and vulva. Clin Obstet Gynecol 1969;12:198-218.

3 Eschenbach DA. Vaginal infection. Clin Obstet Gynecol 1983;26:186-202.

4 Anthony BF, Eisenstadt R, Carter J, et al. Genital and intestinal carriage of group B streptococci during pregnancy. $J$ Infect Dis 1981;143:761-6.

5 Baker CJ, Goroff DK, Apert S, et al. Vaginal colonisation with group B streptococcus: A study in college women. $J$ with group B streptococcus:

6 Bagdade JD, Root RK, Dulgar RJ. Impaired leucocyte function in patients with poorly controlled diabetes. Diabetes 1974;23:9-15. 